\title{
Kirigami Haptic Swatches: Design Methods for Cut-and-Fold Haptic Feedback Mechanisms
}

\author{
Zekun Chang $^{1}$, Tung D. Ta ${ }^{1}$, Koya Narumi ${ }^{1}$, Heeju Kim ${ }^{1}$, Fuminori Okuya ${ }^{1}$, Dongchi Li ${ }^{1}$, \\ Kunihiro Kato ${ }^{1}$, Jie Qi ${ }^{1}$, Yoshinobu Miyamoto ${ }^{2}$, Kazuya Saito ${ }^{3}$, Yoshihiro Kawahara ${ }^{1}$ \\ ${ }^{1}$ The University of Tokyo ${ }^{2}$ Aichi Institute of Technology ${ }^{3}$ Kyushu University \\ \{zekun.chang, tung, narumi, okuya23, leedongchi,jie, kawahara\}@akg.t.u-tokyo.ac.jp, \\ Heeju.kim@network.rca.ac.uk, kkunihir@acm.org, yoshinobu.miyamoto@gmail.com, \\ k-saito@design.kyushu-u.ac.jp
}
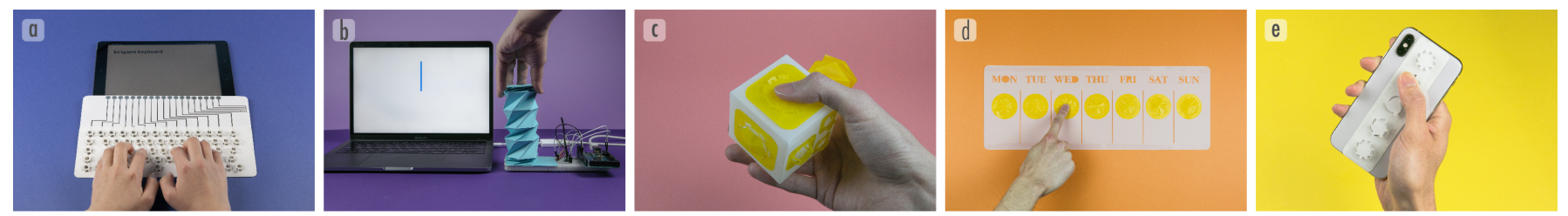

Figure 1: Applications that are designed based on the Kirigami Haptic Swatches. (a) a custom keyboard input interface, (b) a rotational switch, (c) a multi-sensory toy, (d) a task checklist, (e) phone accessories.

\begin{abstract}
Kirigami Haptic Swatches demonstrate how kirigami and origami based structures enable sophisticated haptic feedback through simple cut-and-fold fabrication techniques. We leverage four types of geometric patterns: rotational erection system (RES), split-fold waterbomb (SFWB), the overlaid structure of SFWB and RES (SFWB+RES), and cylindrical origami, to render different sets of haptic feedback (i.e. linear, bistable, bouncing snap-through, and rotational force behaviors, respectively). In each structure, not only the form factor but also the force feedback properties can be tuned through geometric parameters. We experimentally analyzed and modeled the structures, and implemented software to automatically generate $2 \mathrm{D}$ patterns for desired haptic properties. We also demonstrate five example applications including an assistive custom keyboard, rotational switch, multi-sensory toy, task checklist, and phone accessories. We believe the Kirigami Haptic Swatches helps tinkerers, designers, and even researchers to create interactions that enrich our haptic experience.
\end{abstract}

\section{Author Keywords}

Kirigami structure; haptics; paper button; design methods; computational fabrication.

\footnotetext{
Permission to make digital or hard copies of all or part of this work for personal or classroom use is granted without fee provided that copies are not made or distributed for profit or commercial advantage and that copies bear this notice and the full citation on the first page. Copyrights for components of this work owned by others than ACM must be honored. Abstracting with credit is permitted. To copy otherwise, or republish, to post on servers or to redistribute to lists, requires prior specific permission and/or a fee. Request permissions from permissions@acm.org.

CHI '20, April 25-30, 2020, Honolulu, HI, USA.

(C) 2020 Association for Computing Machinery.

ACM ISBN 978-1-4503-6708-0/20/04 ...\$15.00.

http://dx.doi.org/10.1145/3313831.3376655
}

CCS Concepts

-Human-centered computing $\rightarrow$ Haptic devices; User interface toolkits;

\section{INTRODUCTION}

The sense of touch is one of the most fundamental perceptions used for human interaction in the physical world. Touch - and the resulting haptic feedback - helps us understand the objects around us, navigate in and around our environments, and communicate with others in our shared spaces. While touch is present within every single interaction with the physical world, we still observe that many of the everyday haptic interactions with the digital world are largely mediated through the simple pushbutton [16]. However, physical buttons can potentially provide more sophisticated haptic feedback during their travel range, which is commonly described in the form of force-displacement curves [15, 18]. Ubiquitous from early electronics lab prototypes all the way to polished off-the-shelf consumer electronics products, the force characteristics of the physical button play an important role in defining our haptic experience with our digital environments.

The haptic properties of a physical button are determined by its mechanical structure and material. Off-the-shelf buttons such as membrane-dome buttons and buckling-spring buttons have their force-displacement curves fixed during fabrication and only exist in standardized packages with standardized mechanical characteristics. As a result, engineers and designers are typically limited to designing haptic interactions that fall within the parameters of these pre-determined, massmanufactured electronic components. Instead, we aim to enable designers and engineers to easily create their own buttons 
based on desired haptic properties by blending kirigami and origami techniques with paper-based electronics and computationally generated design.

The Kirigami Haptic Swatches presents an approach to easily design and fabricate buttons with customizable haptic feedback based on common kirigami and origami primitives. We leverage four types of geometric cut-and-fold patterns: rotational erection system (RES), split-fold waterbomb (SFWB), the overlaid structure of SFWB and RES (RES+SFWB), and cylindrical origami, to render a variety of haptic feedback interactions (i.e. linear, bistable, snap-through, and rotational force behaviors, respectively). Variations in substrate material and fabrication parameters offer even more variables for tuning the button's haptic properties.

Through mathematical modeling and experimental validation, we created software that generates custom cut-and-fold patterns for paper buttons based on the user's desired form factor and haptic curve profiles. Our kirigami and origami based structures can be made of any flat and foldable sheet—such as paper or PET film - and are assembled using a variety of simple cut-and-fold techniques. Likewise, our user interface is built upon software popular in design communities. By using inexpensive and commonly available substrate materials, widely accessible tools and software, and simple 2D fabrication methods, we designed the Kirigami Haptic Swatches to be an accessible yet powerful way for designers, engineers, and makers of all backgrounds to create customized haptic interactions.

The key contributions of this paper are as follows:

1. Design of haptic feedback buttons based on cut-and-fold fabrication: We present four types of kirigami and origami button primitives that enable four types of haptic feedback profiles

2. Model and analysis: We experimentally analyzed and mathematically modeled the force feedback behaviors of the four haptic mechanisms

3. Pattern generator: We implemented software that uses our mathematical models to generate custom cut-and-fold button patterns based on users' desired strength, feel, and form factor

4. Example applications for Kirigami Haptic Swatches buttons: We explore a variety of scenarios that use our custom kirigami buttons including an assistive custom keyboard, rotational switch, multi-sensory toys for training the sense of touch, task checklist, and accessories for stress release as shown in Figure 1.

\section{RELATED WORKS}

Customizing the haptic qualities of physical buttons is a challenge addressed by researchers through a variety of approaches. The following related works share some of these methods which the Kirigami Haptic Swatches build upon, from making the button itself easier to fabricate through do-it-yourself (DIY) approaches, to new ways to create dynamically-tunable buttons.

\section{Rapid Fabrication of Physical Buttons}

In Pushables [16], Klamka et al. proposed a DIY fabrication approach to prototype membrane buttons with clicking effects by applying heat to the plastic film and embossing it into a dome shape. While this approach makes fabrication easier, it does not discuss tunability of the button's haptic properties, which makes it hard for users to customize buttons depending on their needs. Other groups rendered tactile feedback with printed components; for example, Frisson et al. proposed rendering vibrotactile sensation with printed piezoelectric actuators [3]. Kato et al. and Groeger et al. have recently used inkjet printed circuits on paper substrates to prototype objects with electrostatic force sensation both in 2D [12] and 3D shape [4], respectively. Despite relatively easier print-based fabrication, however, such methods only create tactile sensations by generating signals on the surface of human skin, thus lacking the more kinesthetic sensations and design affordances of physical buttons.

\section{Kirigami and Origami based Structure}

Origami is the art of making 2D/3D shapes by folding paper, and kirigami is an extended method of origami by allowing cuts in addition to folds. Kirigami and origami based structures are particularly inspiring to engineers and researchers in human-computer interaction (HCI). Origami is recently drawing attention in and applied to the field of $4 \mathrm{D}$ printing [1, 6], where a 2D sheet is transformed into a 3D structure after fabrication driven by certain stimuli (e.g., heat). In this context, the biggest benefit of origami is how it enables easy and rapid fabrication of complicated 3D shapes out of diverse and accessible tools such as a 3D printer, a cutting plotter, a laser cutter, or even hand-held scissors. In this paper, rather than focusing on the initial and final shape of the origami before/after folding transformation, we leverage the mechanical properties of origami and kirigami during the folding transformation as a way to easily prototype haptic buttons.

\section{Paper Circuits}

The HCI community has long been paying great attention to paper-based electronics including both hand-made $[8,21,24]$ and digitally printed methods $[5,11,13,14]$. For example, Wessely et al. proposed interactively designing digital objects by cutting the paper with scissors, in which the circuit pattern on the paper substrate can detect its own shape by capacitance change [26]. These techniques for paper circuits have enabled not only professional engineers and researchers but also designers, makers, and even children to prototype and build functional electric components. Our Kirigami Haptic Swatches are compatible with these paper electronics as mechanical, haptic components, since both of them are implemented on paper substrates. We will demonstrate how electronics and kirigami haptic buttons are integrated in the application section.

\section{Haptic Feedback Control by Mathematical Formulation}

In order to control haptic sensations of the interactive systems, researchers have analyzed and utilized mathematical relationships between physical parameter pairs. For example, Liao et al. has recently reported four types of force-displacement curves of a single kinesthetic pin [18] to simulate different 
haptic effects of conventional keyboards. Also, Kim et al. controls the force-displacement curve of a single tactile actuator to build a virtual button with different clicking effects [15]. In a different approach, Ogata controls the potential energy of magnetic fields against displacement, by distributing multiple permanent magnets to build a linear slider with diverse kinesthetic motion [22]. Nakagaki et al. built upon pin display techniques in HCI $[2,9,10]$ and presented Materiable [20] which can render several rheological properties of materials such as elasticity and viscosity, using the relationship between displacement and time. Among these types of different formulations, we utilize the force-displacement curve in this paper.

\section{Uniqueness of our Technique}

Our technique is unique from the previous works cited in terms of (1) easy and simple origami/kirigami fabrication method using accessible sheet materials and maker-friendly tools, (2) controllable force-displacement properties, and (3) compact and untethered implementation that works without external energy sources (e.g., electricity, rigid motors, pneumatic force).

\section{OVERVIEW OF KIRIGAMI HAPTIC SWATCHES}

Kirigami Haptic Swatches are comprised of four kirigami and origami primitives for haptic feedback. Figure 2 gives an overview of the structure, mechanical motion, and the typical force-displacement curve that each primitive offers.

Rotational erection system (RES) has a linear property; as you push or release the structure, it moves accordingly without a click. Split-fold waterbomb (SFWB) has a bistable property; if you apply more force than a certain threshold, the structure suddenly buckles, resulting in a clicking feeling and does not bounce back even after releasing the applied force. The overlaid structure of SFWB and RES (SFWB+RES) has a snap-through property that generates a click like SFWB, but bounces back with another click if the user releases the applied force. Finally, cylindrical origami shows bistability like SFWB, by buckling with a click with no bouncing back; the difference between the cylindrical origami versus all the other primitives is that this structure exhibits angular rotation when force is applied. Table 1 summarizes comparisons between these four primitives.

In the next sections, we will describe how users can apply the Kirigami Haptic Swatches to making buttons using the Pattern Generator, provide an in-depth mathematical and experimental analysis of each primitive structure, and share example applications of Kirigami Haptic Swatches.

Table 1: Comparison of four primitives

\begin{tabular}{l|c|c|l} 
Primitive & Click & Bounce back & Direction \\
\hline \hline RES & No & Yes & Straight \\
SFWB & Yes & No & Straight \\
SFWB+RES & Yes & Yes & Straight \\
Cylindrical origami & Yes & No & Rotational
\end{tabular}

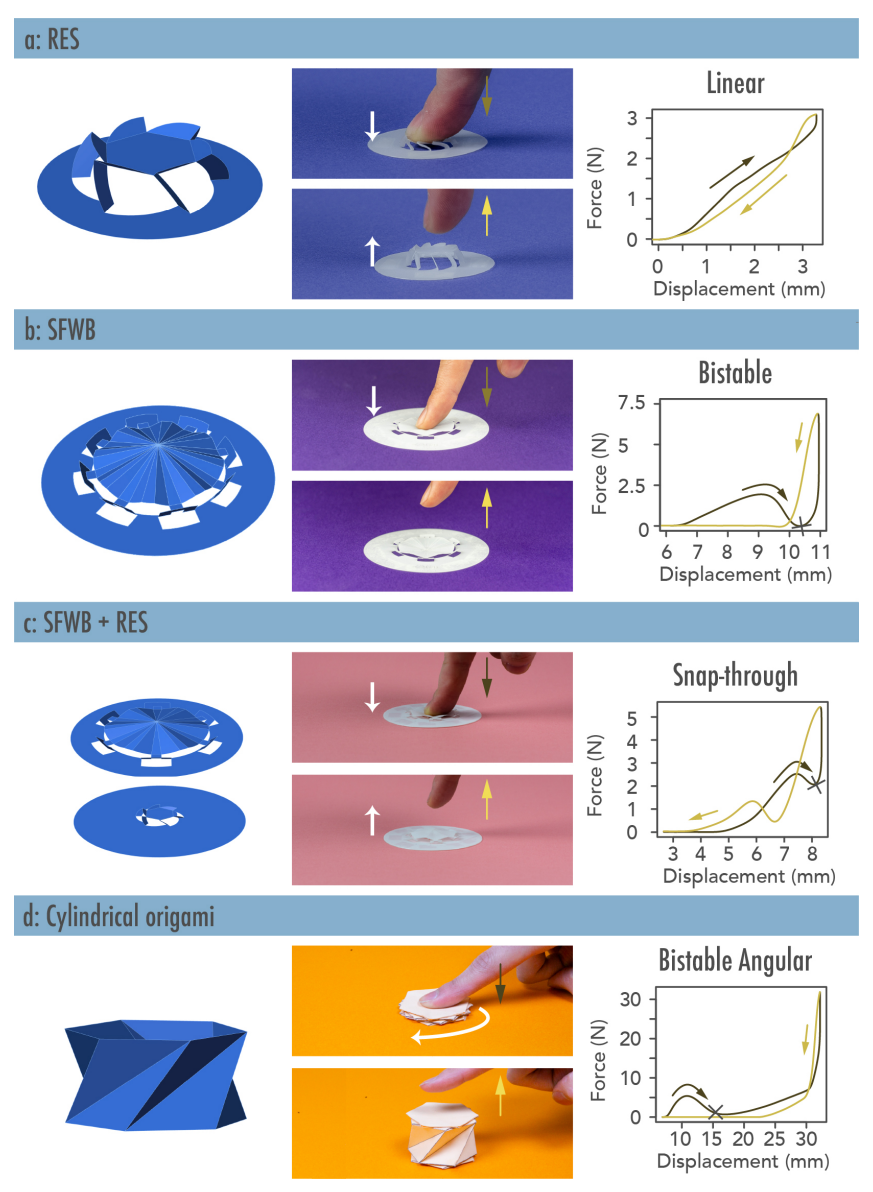

Figure 2: Four primitives of Kirigami Haptic Swatches. Each row includes a model of the structure, photos of press and release motion, and typical force-displacement curve from experiments. " $X$ " in the graphs show the points with click feeling. (a) RES has a linear property. (b) SFWB has a bistable property. (c) SFWB+RES has a snap-through property. (d) cylindrical origami has a bistable and angular property.

\section{PATTERN GENERATOR}

To make the Kirigami Haptic Swatches accessible to everyday creators, we made a plugin software that generates custom cutand-fold patterns for buttons based on users' desired haptic feedback properties.

\section{Generator Software}

The Pattern Generator plugin is made for Rhinoceros 6 and Grasshopper, and it is available on Github ${ }^{1}$.

The software interface, shown in Figure 3, allows users to choose among the four primitive structures: RES, SFWB, RES+SFWB, and Cylindrical Origami. For each primitive structure selection, users can input the form factor variables of the button (e.g. diameter, key-press traveling distance, maximum button height) and the software will calculate the maximum reaction force. Likewise, users can also input the

\footnotetext{
${ }^{1}$ https://github.com/KawaharaLab/Kirigami-Haptic-SwatchesPattern-Generator
} 


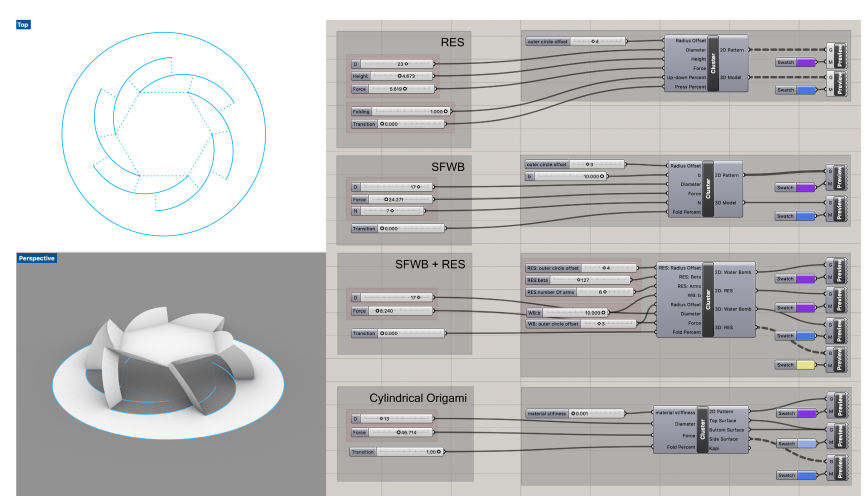

Figure 3: Pattern Generator interface showing example 2D kirigami (top left), 3D model (bottom left) and input parameters (right).

desired maximum reaction force and the software will generate the corresponding geometric parameters. Due to differences in geometric structure, available parameters differ depending on which primitive is selected.

Based on these design parameters our software generates the corresponding 2D kirigami cut-and-fold pattern (automatically selecting parameters like number of arms and density of perforations scoring fold lines) and a 3D model preview of the assembled button in real time, allowing users to see a live rendering of their design.

\section{Using the Pattern Generator}

Users must first decide which base primitive to use based on what kind of force profile (shown in Table 1) they need for their application. Next, they input design parameter values and iterate until the input values achieve the target form factor and force profile.

Once the desired design is completed, the user can download the output 2D kirigami pattern in a standard 2D file format (such as .ai, .dxf, or .dwg) and transfer this to a laser cutter or cutting plotter for digital fabrication. If these tools are not available, the templates can also be simply printed out on a traditional printer and cut using scissors or a cardboard cutter.

Finally, once the template is cut, users fold the patterns by hand to assemble the completed mechanical button. Complexity of assembling the buttons depends on the button type. For RES, users simply twist the center of the button. For buttons incorporating the SFWB, users need to make a series of mountain and valley folds to create the central hub. For the cylindrical origami, users also need to tape or glue the ends of the paper to create the final cylindrical tube.

\section{MODELS AND ANALYSES OF KIRIGAMI PRIMITIVES}

In this section, we present the mathematical models and experimentally derived force-displacement curves for the four kirigami primitives that are used in the Pattern Generator.

\section{Experimental Setup}

For each primitive type, we ran a series of force-displacement tests to derive equations connecting geometric parameters with resulting force profiles. In addition, for each primitive type we also ran a fatigue test of 100 presses of one model. For more detail on experimental setup, please see auxiliary materials.

\section{Rotational Erection System (RES)}

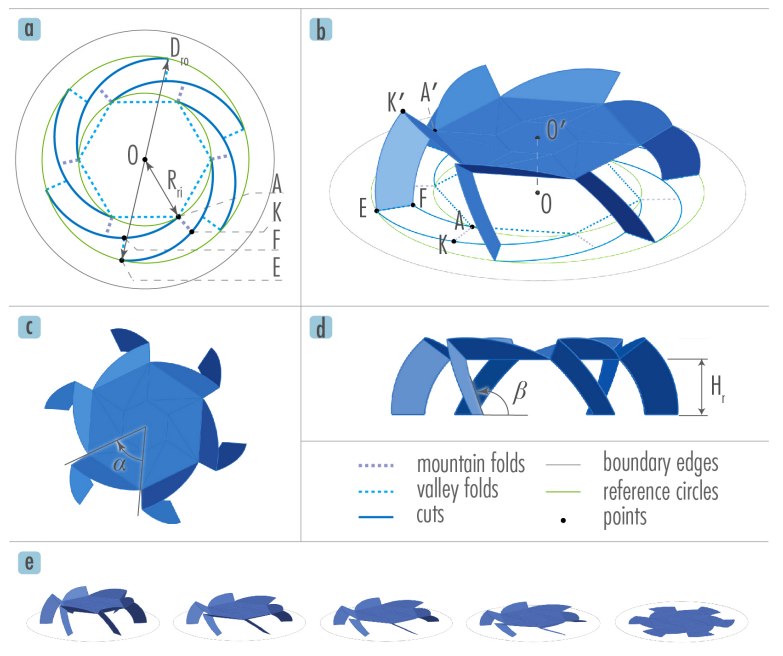

Figure 4: Structure of an RES button. (a) a 2D kirigami pattern of a 6-arm RES button, (b) a 3D popped-up model of the RES button, (c) top view of the RES button, (d) side view of the RES button, (e) transition of a RES button from the erected state to flattened the state.

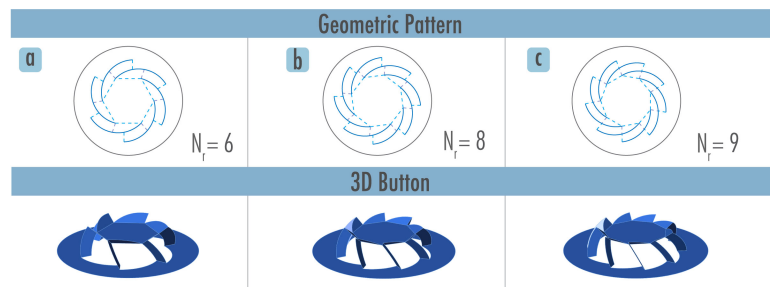

Figure 5: 3D RES buttons from 2D kirigami patterns. (a) an RES button with 6-arms, (b) an RES button with 8-arms, (c) an RES button with 9-arms.

The Rotational Erection System (RES) [19] is used to create a button with a linear force-displacement curve. As users press down on the button, it increases the resistance force linearly, and when users release the applied force the button bounces back. RES is a kirigami pop up technique in which the $2 \mathrm{D}$ sheet is cut with a rotationally symmetric pattern and material is rotated to create a 3D button. Figure 4a shows the RES cuts-and-fold pattern in the 2D flat state, and Figure 4b shows the $3 \mathrm{D}$ popped-up state.

Three features make RES particularly appropriate for building mechanical buttons. First, RES structures reliably reach a single stable state when assembled in three-dimensional form. Second, unlike most other pop up techniques which must actuate based on a central fold-such as in typical greeting cards-RES buttons can rise out of the base structure and move independently without interfering with or depending on neighboring mechanisms. This allows designers to create 
multiple buttons on one substrate sheet. Finally, the RES cut pattern frees the RES button material from surrounding material. This makes it much easier for designers to create cutand-fold patterns with multiple buttons as they do not need to worry about the material constraints of neighboring structures typically associated with tessellated origami patterns.

Geometric Parameters for Form Factor

$$
H_{r}=\frac{2 R_{r i} \sin \left(\alpha_{r} / 2\right) \sin \beta_{r}}{1-\cos \beta_{r}}
$$

Angle $\alpha_{r}$ is the horizontal rotation angle and angle $\beta_{r}$ is the vertical rotation angle defined by the difference between the flat pattern and the erected structure. $N_{r}$ is the total numbers of arms in the RES button. The diameter $D_{r o}=2 \mathrm{OE}$ of the outer reference circle and the radius $R_{r i}=\mathrm{OA}$ of the inner reference circle determine the size of the button and button cap. The height $H_{r}$ of the button is calculated through Eq. 1. We note that we used $\alpha=60^{\circ}, \mathrm{OA}=0.62 \mathrm{OE}$, and $\mathrm{AK}=\mathrm{EF}$ for simplicity of design.

\section{Geometric Parameters for Force Behaviors}

In order to analyze the behaviors of the RES, we tested the force-displacement with different geometric parameters set $\mathrm{OE}=10 \mathrm{~mm}, N_{r}=\{3,6,9\}, D_{r o}=$ $\{14 \mathrm{~mm}, 20 \mathrm{~mm}, 26 \mathrm{~mm}\}, \beta_{r}=\left\{120^{\circ}, 135^{\circ}, 150^{\circ}\right\}$, and ppi $_{r}=\{92,145,399\}$. A linear regression was calculated to predict maximum reaction force $F_{\text {rmax }}$ based on geometric parameters $N_{r}, D_{r o}, \beta_{r}$, and $p p i_{r}$. The predicted maximum force is calculated as shown in Eq. 2 with $R^{2}=0.89$.

$$
F_{\text {rmax }}=0.37 N_{r}+0.07 D_{\text {ro }}+0.01 \beta_{r}-0.005 p p i_{r}+0.9
$$
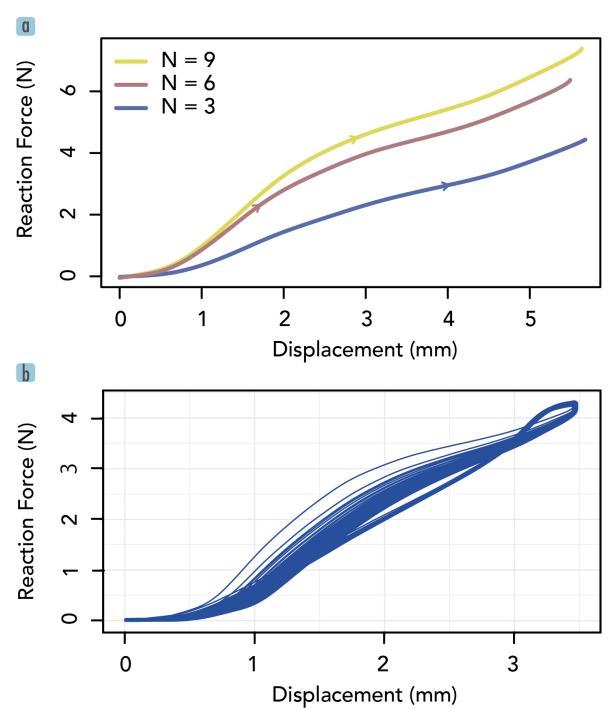

Figure 6: Force-displacement curve of RES, derived from experiments with physical samples. (a) loading curve of RES with three different parameters of $N$ (median value is shown, $n=50$ ). (b) fatigue test of RES with 100 times loadingunloading cycles showing the good mechanical durability of RES.
Figure 6a demonstrates the linear force-displacement curve of $\operatorname{RES}\left(N_{r}=\{3,6,9\}, \mathrm{OE}=13 \mathrm{~mm}, \beta_{r}=135^{\circ}\right.$, and $\left.p p i_{r}=92\right)$, in which resistance of the RES button continuously increases with the key press. The keystroke is a straight downward drop with no perceptible bump or click. The hysteresis of releasing the button also shows linearity of the reaction force (as shown in Figure 2a). Buttons with such linear haptic feedback properties are often coveted for their quiet, smooth action as well as how their force feedback curves are independent of interference from the interaction itself.

The fatigue test result in Figure $6 \mathrm{~b}$ shows that the RES is resilient and durable under repeated loading-unloading cycles. There is deviation among cycles, but the hysteresis shape and maximum reaction force are stable.

\section{Split-fold Waterbomb (SFWB)}

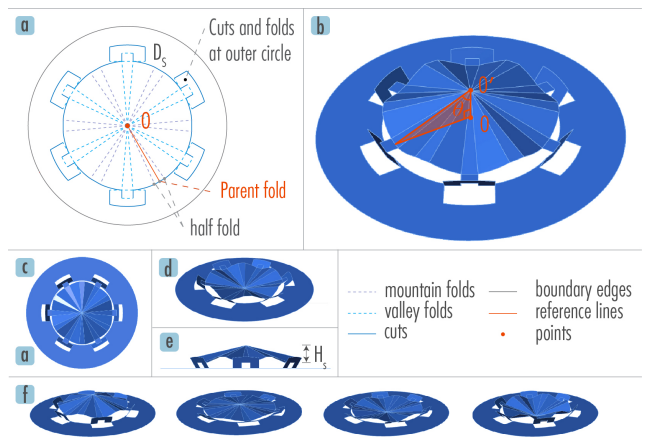

Figure 7: Structure of a SFWB button. (a) a 2D kirigami pattern of a 6-arm SFWB button, (b) a 3D convexly poppedup model of the SFWB button, (c) top view of the SFWB button, (d) a 3D concavely popped-down (= buckled) model of the SFWB button, (e) side view of the SFWB button, (f) transition of a SFWB button from popped-up to popped-down state.

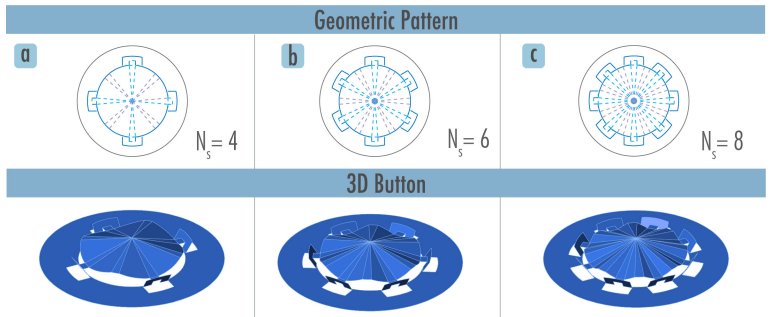

Figure 8: 3D SFWB buttons from 2D kirigami patterns. (a) an SFWB button with 4 parent mountain folds, (b) an SFWB button with 6 parent mountain folds, (c) an SFWB button with 8 parent mountain folds.

A bistable button is made using a split-fold waterbomb (SFWB) pattern [7] with extended cuts-and-folds on the outer circle (as shown in Figure 7a). When users press this bistable button, it has a clicking feeling but does not bounce back to the original position.

The traditional origami waterbomb (WB) pattern is four valley folds alternating with four mountain folds on a square sheet. 
The generalized origami WB is a single-vertex structure with an even number of alternating mountain and valley folds that can be folded and moved without deflecting neighboring facets. As illustrated in Figure 7, the SFWB is made by splitting each fold of a general WB into two "half folds" [7] of the same variety as the parent fold. In our design, we split the parent fold into two "half folds" which constitute an angle of $10^{\circ}$ with the parent fold as bisector (as shown in Figure 7a).

The SFWB structure naturally is a nonlinear spring with bistable properties, making it appropriate for creating bistable haptic buttons. By adjusting the geometry of cuts and folds, we are able to tune the force feedback as well as form factor of the button. Furthermore, we design additional cuts-and-folds on the outer circle (Figure 7a) that connects the SFWB to the surrounding flat plane, thus isolating each SFWB button from the surrounding material and enabling localized manipulation.

\section{Geometric Parameters for Form Factor}

The alternating split mountain "half folds" and split valley "half folds" are the primary elements of an SFWB button. $N_{s}$ is the number of parent mountain folds (or valley folds) for an SFWB button. Angle $\theta$ is the angle measured between any center line of a valley split-fold facet and the vertical axis that passes through the vertex perpendicular to the flat plane. $D_{s}$ is the diameter of SFWB that defines the size of the SFWB button. The height $H_{S}$ of SFWB mechanism, which defines the travel distance of an SFWB button, is calculated through Eq. 3.

$$
H_{s}=\frac{1}{2} D_{s} \cos \theta
$$

To simplify the model, the length of mountain folds and valley folds are made equal so that the mechanism becomes symmetric.

The diameter $D_{s}$, height $H_{s}$ and number of parent mountain (or valley) folds $N_{s}$ are used to generate the geometric cut-and-fold patterns of an SFWB button.

\section{Geometric Parameters for Force behaviors}

In this configuration, $N_{s}, H_{s}$, and $D_{s}$ are the three key parameters that affect the force behaviors of SFWB buttons. The number of parent mountain folds $N_{s}$ in the SFWB pattern is the dominant factor for tuning the reaction force (Figure 8). The more creases there are, the more potential energy is stored in the mechanism, and thus the greater the force needed to press and flip the state of the button. As button diameter $D_{s}$ increases, the total length of the hinges increases, which results in an increase in the reaction force of the SFWB when pressed. Increasing the height $H_{s}$ results in an increase in the range of motion of each fold, thus increasing the force required to flip the button. The analytic model between reaction force and geometric parameters of the SFWB was thoroughly investigated in [7]. We set up the SFWB experiment with different values of $p p i$ and measured the reaction forces. By mapping the measured reaction force and the analytically calculated reaction forces in Eq.(18) of [7], we were able to derive the relationship between $K$ and $p p i$ for the PET film: $K=-0.022 \times p p i+8.777$, where $K=k_{\gamma m}=k_{\gamma v}$ represents the stiffness of mountain and valley folds.
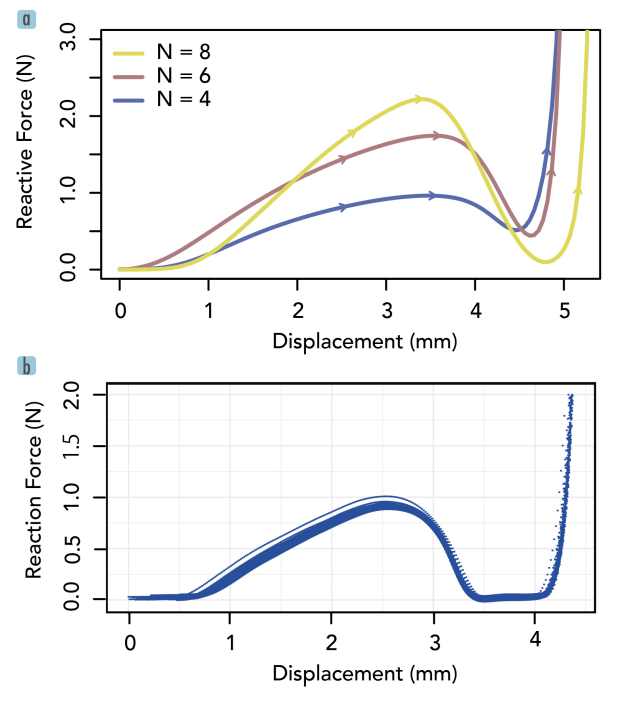

Figure 9: Force-displacement curve of an SFWB button, derived from experiments with physical samples. (a) loading curve of an SFWB button with three different parameters of $N$ (median value is shown, $n=50$ ). (b) Fatigue test of an SFWB button with 100 times loading-unloading cycles showing the good mechanical durability of SFWB.

\section{Experimental Setup and Results}

Figure 9a plots the bistable force-displacement curve of the SFWB button $\left(N_{s}=\{4,6,8\}, D_{s}=30 \mathrm{~mm}, p p i_{s}=50\right)$, showing two stable points where the button rests and force is at a local minimum. The fatigue test in Figure 9b show the stability of the SFWB bustton. We also see here there is a plateau valley after the button pop down. The reason is that the load cell of the force tester was not in contact to the button after it pops.

\section{Overlaid Structure of SFWB and RES (SFWB+RES)}

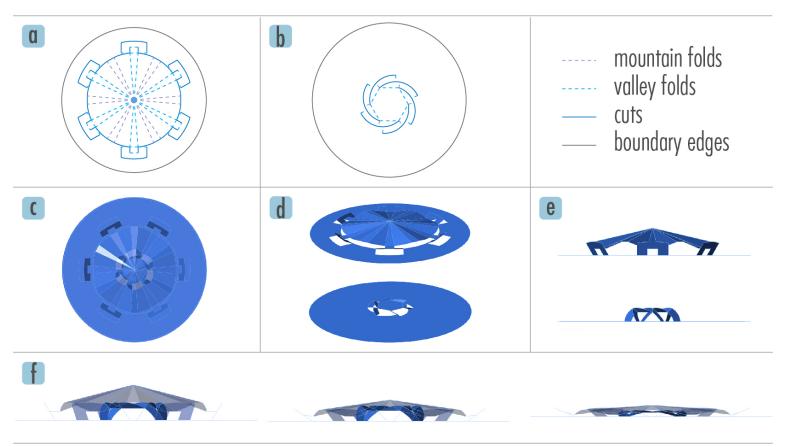

Figure 10: Structure of an SFWB+RES button. The bottom layer RES is overlaid by the top layer SFWB. (a) Kirigami pattern of an SFWB, (b) Kirigami pattern of a RES, (c) top view of the SFWB overlaying the RES, (d) 3D view of the overlay, (e) side view of the overlay, (f) Transition of the RES+SFWB from the popped-up state to the pressed-down state. The structure bounces back after pressed down. 

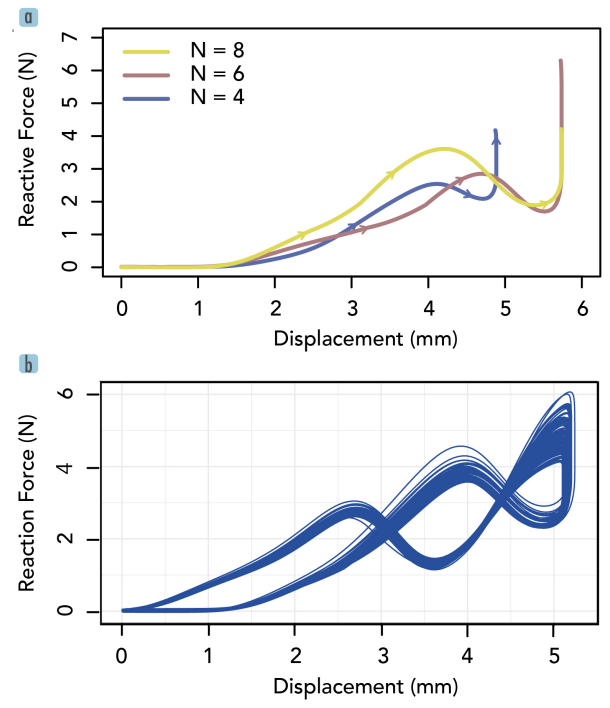

Figure 11: Force-displacement curve of SFWB+RES, derived from experiments with physical samples. (a) loading curve of SFWB+RES with three different parameters of $N$ (median value is shown, $n=50$ ). The clicking motion of an SFWB+RES button is generated by the SFWB layer, but the existence of the RES allows the whole structure to bounce back, resulting in snapping through motion. (b) fatigue test of an SFWB+RES button with 100 times loading-unloading cycles showing the good mechanical durability of SFWB+RES.

We overlaid the SFWB and RES (SFWB+RES) mechanisms to create a button with mono-stable snap-through forcedisplacement properties. The SFWB layer serves as the "clicking" factor while the RES layer is used as a linear "spring" to provide the returning force. This way, the button returns to the original position once the user releases the button.

\section{Geometric Parameters for Form Factor}

As illustrated in Figure 10, the primary elements of a RES+SFWB structure include a RES in the bottom layer and an SFWB in the top layer. The form factor of a RES+SFWB button is largely determined by the SFWB cap structure, i.e. the diameter $D_{s}$ and the height $H_{s}$ determine button size and travel distance. The diameter $D_{r o}$ of the RES layer just needs to be smaller than the diameter $D_{s}$ of the SFWB layer in order to fit.

\section{Geometric Parameters for Force behaviors}

The force feedback characteristics of RES+SFWB buttons largely depend on the outer SFWB structure. Therefore, $N_{s}$, $H_{s}$, and $D_{s}$ are the three key parameters that affect the force behaviors of RES+SFWB buttons. By varying the $N_{s}, H_{s}$, and $D_{s}$, the force behaviors change similar to that of SFWB-only buttons.

\section{Experimental Setup and Results}

Figure 11a shows the monostable force-displacement curve with snap-through behavior of the RES+SFWB button. Here, we overlaid the SFWB buttons from the previous section $\left(N_{s}=\{4,6,8\}, D_{s}=30 \mathrm{~mm}, p_{p i}=50\right)$ on top of an 6-arm
$\operatorname{RES}\left(N_{r}=6, D_{r}=14 \mathrm{~mm}, \beta_{r}=150^{\circ}\right.$, and $\left.p p i_{r}=399\right)$. The reaction force rises along with the press of the button up to a maximum value followed by a sudden decrease in force when the button snaps. Upon release the RES layer pushes the structure back to the position which the SFWB will snap back up the original stable, unpressed state. Due to the underneath RES, the force-displacement curves does not come close to horizontal axis as that of the single SFWB in Figure 9a. The hysteresis of the combined button is shown in Figure $2 \mathrm{c}$ where there is also a snap back moment which provides haptic during unloading. This force-displacement curve is attractive because it creates the same types of haptic feedback commonly found in commercially available electronic devices.

\section{Cylindrical Origami}

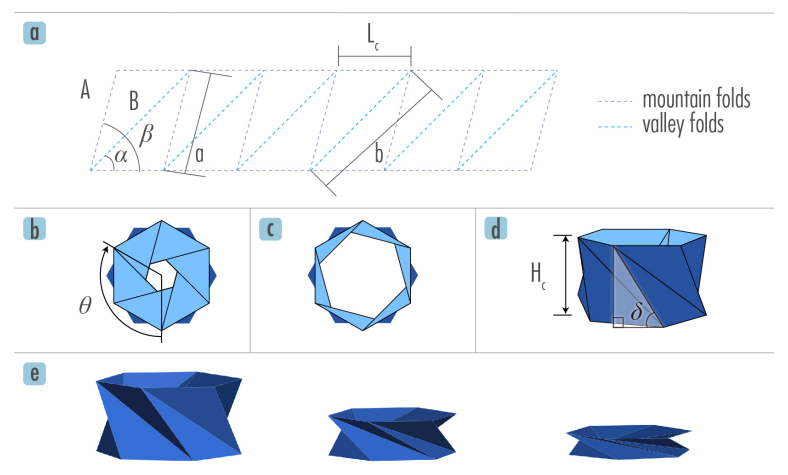

Figure 12: The structure of a cylindrical origami button. (a) the crease pattern of a cylindrical origami unit. The topview in the closed state (b) and open state (c). (d) The sideview. (e) transition of a cylindrical origami, showing rotation and bistable buckling at the same time.

Cylindrical origami [27] is a technique to create a cylinder made up of smaller stacked cylindrical units folded using the Kresling pattern [17]. Each cylindrical sub-unit exhibits rotational and bi-stable force behaviors, allowing us to make buttons with multi-stable haptic properties. As users press on the button, they can experience multiple "clicking" sensations along with rotational force feedback.

\section{Geometric Parameters for Form Factor}

Since cylindrical origami can be made up of an arbitrary number of stacked sub-units, our model looks at the properties of a single cylindrical unit first. Let $L_{c}$ be the length of a side of the regular hexagon, $\alpha_{c}$ and $\beta_{c}$ are the angles of the crease pattern (as illustrated in Figure 12). According to the analysis in [25], the transformation between two bistable states is largest when $\beta_{c}-\alpha_{c}=30^{\circ}$. The height of each unit can be calculated through the Eq. 4 [23].

$$
H_{c_{\text {unit }}}=L_{c} \sqrt{2 \sqrt{3} \sin \left(2 \alpha_{c}-60^{\circ}\right)}
$$

\section{Geometric Parameters for Force Behaviors}

The cylindrical origami button is designed to render multistable force behaviors with rotational motions. Each cylindrical unit is a bistable structure, which is stable at both the expanded and compressed states. Let $F_{\text {trans }}$ be the required 

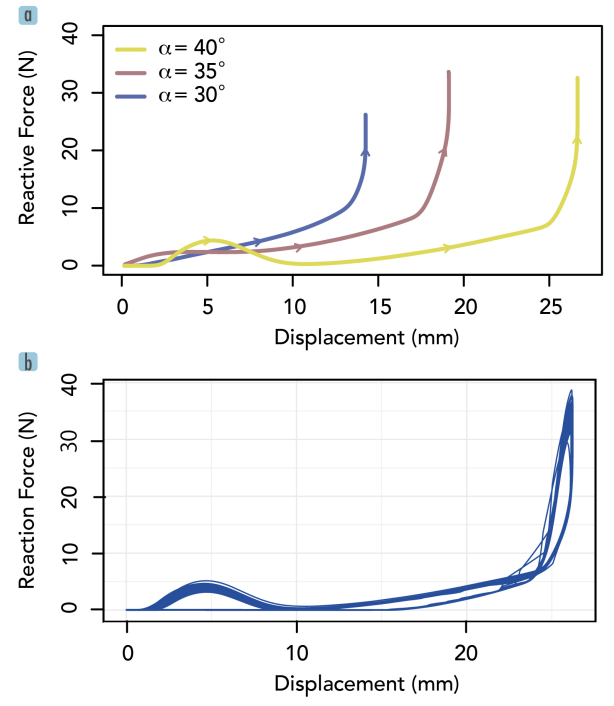

Figure 13: Force-displacement curve of a cylindrical origami button. (a) loading curve of a cylindrical origami with three different parameters of $\alpha$ (median value is shown, $n=50$ ). (b) fatigue test of a cylindrical origami button with 100 times loading-unloading cycles showing the good mechanical durability.

force to enable the switch between two bistable states. In the case of a diameter $D_{c}$ hexagon cylindrical origami as in Kirigami Haptic Swatches, let $\delta_{c}=\alpha_{c}-30^{\circ}$ and $K_{c}$ be the material stiffness, the transitional force can be calculated as in Eq. 5.

$$
F_{\text {trans }}=K_{c} D_{c}^{2}\left(0.062 \delta_{c}^{3}+0.0645 \delta_{c}^{2}-0.4388 \delta_{c}\right) \times 10^{-5}
$$

The value of $F_{\text {trans }}$ for state transformation can be adjusted by changing the crease pattern angle $\alpha_{c}$. The greater the $\alpha_{c}$ of a cylindrical unit, the more force $F_{\text {trans }}$ is required to shift the unit's state. As force is applied to the cylindrical button, units with the lower $\alpha_{c}$ will buckle earlier than units with higher $\alpha_{c}$. This property enables the cylindrical origami button to be compressed unit by unit in a controllable sequence, giving users multiple clicking sensations as well as twisting haptic feedback.

We used Takeo tant paper to make 1-unit cylindrical origami buttons with geometric parameters as $\alpha_{c}=\left\{30^{\circ}, 35^{\circ}, 40^{\circ}\right\}$ and $L_{c}=30 \mathrm{~mm}$. Figure 13a shows the force-displacement curves of the 1-unit cylindrical origami with a different value of $\alpha_{c}$. It is evidently shown from the graph that a higher $\alpha_{c}=40^{\circ}$ gives better haptic feedback as it requires a stronger force to switch between two bi-stable states. In the case of $\alpha_{c}=35^{\circ}$, there is a slight bistable jumping in the beginning. In contrast, $\alpha_{c}=30^{\circ}$ cylindrical origami does not have any bistable switching region, and therefore it does not provide any bumpy feedback.

\section{EXAMPLE APPLICATIONS}

In this section we present a variety of prototype devices and use scenarios to explore the range of design and interaction possibilities enabled by Kirigami Haptic Swatches buttons.

\section{Custom Keyboard Input Interfaces}

By attaching RES buttons to the inkjet-printed paper circuit, we created several types of kirigami keyboards as assistive input interfaces. Among several design protypes, Figure 14 shows a emoji keyboard, a full keyboard with custom distribution, and a keyboard for mathematicians who frequently use greek letters. Figure 15 describes the mechanism of kirigami keyboard in case of a single RES button. When the users touch the RES button with a hole at the center, the capacitive touch sensing pattern underneath responds to the finger only when they press down the key. Then the touch event is transferred to the touch surface of the tablet and allows extended touch input [13].

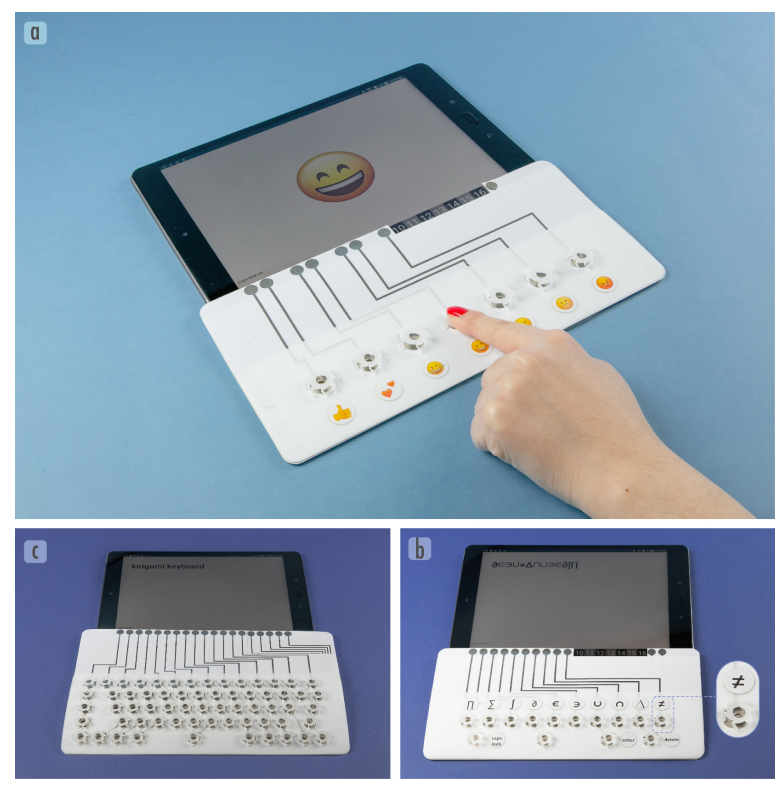

Figure 14: Custom keyboards attached to the tablet. The key-press events are sent via a capacitive touch screen using inkjet-printed conductive patterns. As Examples, an emoji keyboard (a), a full keyboard with custom distribution (b), and a keyboard for mathematicians (c) are demonstrated.

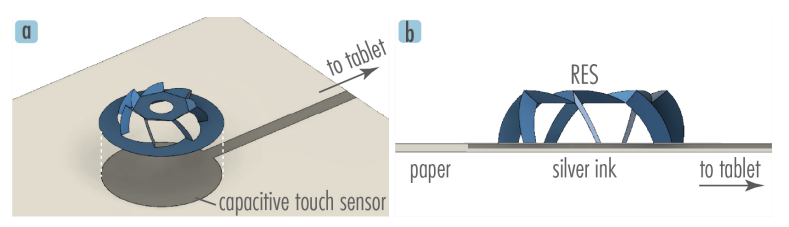

Figure 15: Working principle of a kirigami keyboard. RES structure with a central hole is stacked onto the capacitive touch sensor pattern of paper circuit. Only when the user press down the RES button, the sensor responds and works as a key. $(a, b)$ The perspective and the side view.

\section{Rotational Switch}

We combined a 4-unit cylindrical origami switch and a proximity sensor to measure the length of the cylinder and thus to build a 4-step dial. As shown in Figure 16, the physical rotational motion controls a virtual dial on the display. 

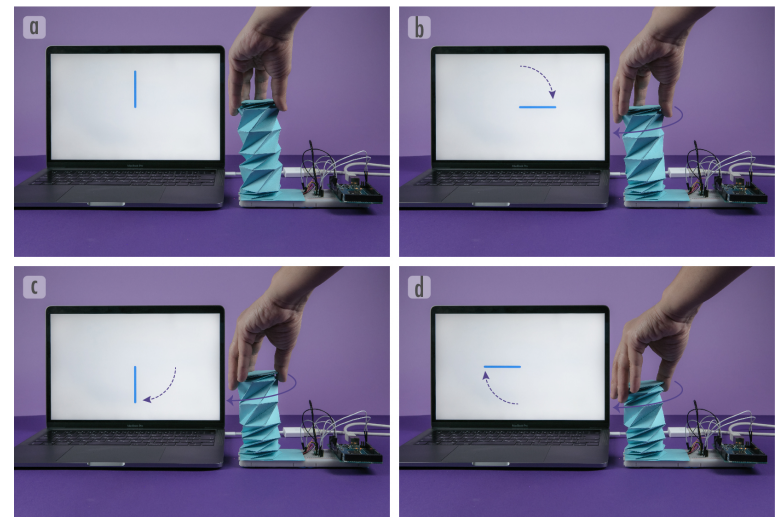

Figure 16: The cylindrical origami structure works as a rotational switch when combined with a proximity sensor.

\section{Multi-sensory Toy}

The multi-sensory toy has different sets of haptic feedback buttons on its surface. By applying different form factor and force properties to six faces of a cube (Figure 17), the multisensory toy aims to help young children who are developing their sense of touch, by providing diverse types of physical touch stimulation and click sounds. Depending on the development stage and needs of the child, we can easily implement a new toy, thanks to easy fabrication.
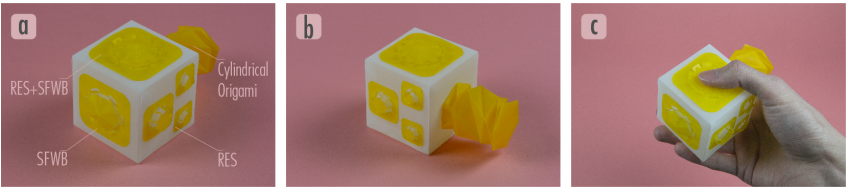

Figure 17: The multi-sensory toy is a cube with different Kirigami Haptic Buttons at different facets.

\section{Task Checklist}

The tangible and interactive task checklist (Figure 18) was designed with SFWB buttons and simply made by pasting the bistable buttons to any standard paper list. When users completed the tasks, they press the kirigami button located beside each task. Pressed buttons do not return to their original position and remain pressed, signaling the completion of the task. This small hands-on play and key click feedback provides users a satisfying interactive experience to mark moments of completion in their daily routine.

\section{Phone Accessory}

The Kirigami Phone Accessory is a therapeutic tool to help people who are addicted to smartphones by helping them stay away from using their devices. Users can select their preferred touch sensation among the Kirigami Haptic Swatches and simply make and attach the button to the backside of the smartphone or case (Figure 19). Instead of turning on the screen, users can interact with their phones instead by playing with the kirigami button. By making the smartphone an interactive haptic device where physical interaction is decoupled from its digital content, the haptic functionality can help distract
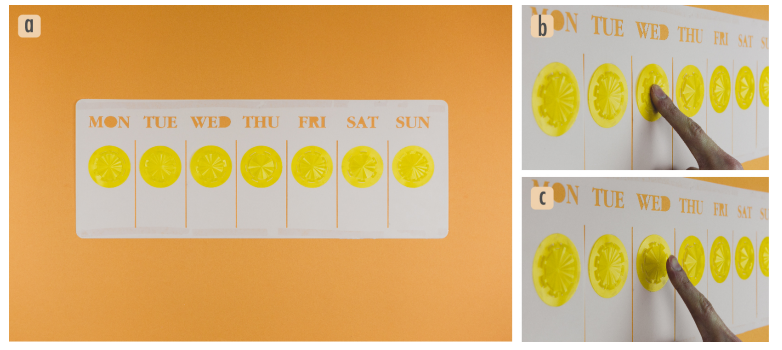

Figure 18: Bistable SFWB buttons are attached to the list of task to make it an tangible and interactive task checklist.

users from addiction as well as reduce stress and tension by physically stimulating the hand as a substitute for phone usage.
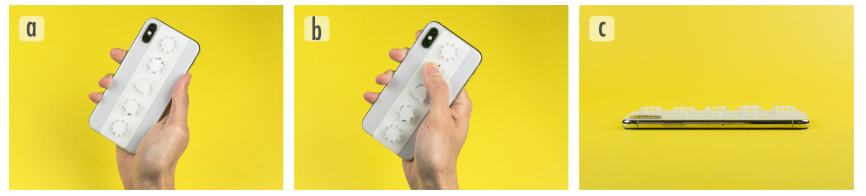

Figure 19: Different haptic RES buttons are attached to the back of a phone to help reducing the phone addiction.

\section{DISCUSSION}

Our goal is to enable designers without engineering backgrounds to design haptic characteristics by controlling desired button parameters themselves though our pattern generator. Rather than needing to choose among only standard buttons, designers now have the opportunity to create their own custom buttons. The cut-and-fold technique also brings accessibility and efficiency to the prototype assembly process, allowing designers to quickly experiment and iterate to achieve their desired effect. This capability provides many benefits and opportunities.

First, without actually testing a standard button, it is often difficult to tell the handfeel-that is the texture and haptic profile or literally how it feels in the hand-of particular buttons. As a result, designers often need to order many different sample buttons in order to find one that achieves approximately their desired effect. This not only adds time to the design process-designers must often wait for delivery of the ordered buttons-it also limits what designers can create, because the desired button may simply not exist or the designer may not have access to purchase the desired component, for example if they are in a geographic location without access to large online or in-person electronics shops. Finally, commercially available buttons with less common haptic profiles also tend to be more costly, making them less accessible to designers with limited resources. Being able to quickly design and create their own buttons using the Kirigami Haptic Swatches thus gives designers a wider range of possibilities, speeds up the design and iteration process and provides a potentially more accessible alternative-substrates like paper and PET sheets are relatively common and affordable materials-to purchasing off-the-shelf button components. 
Being able to customize the haptic experience of buttons also gives designers another dimension to utilize as design vocabulary. The haptic experience can be tuned to match the application context. For example, smaller bistability can contribute to quieter clicks, which may be suitable for use in public, while bigger bistability prevents accidental pushes (e.g., for an alarm clock). Force feedback can also be used to convey meaning or map to information. For instance in the task calendar, more important tasks can require more force to push while in the custom emoji keyboard, the designer iterated until the optimal pushing experience was found to match each emotion. The tangible aspects of the kirigami buttons allows designers to play with the motion of the button as part of the interaction, such as in the twisting switch and multi-sensory toy cube, adding a proprioceptive dimension to the design space. Finally, because the buttons are flat and flexible, they can easily be attached to any flat surfaces (e.g. the back of a phone) for adding interactivity to normally static spaces.

While the Kirigami Haptic Swatches opens many opportunities for designers, it is not without its challenges. The next section shares some limitations and directions for future research.

\section{LIMITATIONS AND FUTURE WORK}

\section{Manual Assembly}

While the pattern generator automates the design of cut-andfold patterns for custom button designs, the final assembly process still requires manual folding. As a result, even though the folding itself is relatively simple, if designers wanted to scale up the number of buttons, the manual assembly process quickly becomes tedious and limiting. Furthermore, as buttons become smaller, the assembly process requires more manual dexterity to successfully fold. This many further limit accessibility for some users.

\section{Fabrication Methods and Consistency}

Currently the folds of our Kirigami Haptic Swatches buttons are scored with laser cut holes making up dotted lines and then folded manually. By varying parameters like the points per inch of the dotted lines or how strongly the material is creased by hand, we can also vary the overall stiffness of a button. Changes to the fabrication process, such as pressing the buttons for an extended period under a heavy weight to "store" the creases, would also affect the force feedback profile of the assembled button. These parameters contribute to some variability in the haptic feedback behaviors of the button. In order to increase consistency between buttons, we can explore creating secondary tools, such as folding tools or jigs to the fabrication process.

\section{Material Variability}

In this paper we have largely focused on geometric parameters for tuning force feedback behaviors of kirigami buttons. However, material properties like stiffness and ductility greatly influence the force feedback response of a given folded material. In future work, we can explore how different types of materials, the same materials with different thicknesses, or composites of multiple materials in laminated sheets might influence the force-displacement curves and enable new haptic feedback capabilities. Furthermore, we have limited our experimental tests to buttons made from PET sheets and only data based on these results power the force calculations in the Pattern Generator. As a result, the Pattern Generator is limited to buttons made from PET sheets while buttons made from other materials may have differing force profiles. We would like to continue developing the Pattern Generator software by collecting data on buttons made from a variety of materials so that we can add these materials profiles to the software.

\section{Durability}

Since Kirigami Haptic Swatches buttons are currently made of soft, hand-foldable materials for ease of fabrication, by definition they are not as sturdy as standard buttons made of tougher materials like rigid plastic and metal. Furthermore, most of our button designs expect a vertical downward force, which works well with the symmetric design of the buttons. However, if users applied an asymmetric shear force to the buttons, this may result in unintended buckling or crumpling of the button structure. While we can explore making more sturdy buttons by using stronger materials, such as sheet metals, this approach would also affect the stiffness of folded hinges and thus the force feedback profile. It may be possible to then counteract these changes by adjusting the geometric parameters, and such adjustments can be informed by further materials explorations.

\section{Geometric Complexity}

Exploring more complex geometries further expands the Kirigami Haptic Swatches. The geometric models presented in this paper are all centrosymmetric and based on the design of the standard push button. However, for example by using an asymmetric cut-and-fold design we can create directional behaviors in the button force feedback profiles. Furthermore, we can explore additional kirigami and origami mechanisms to create swatches patterns for entirely other types of buttons and switches beyond simple push buttons.

\section{CONCLUSION}

In this paper, we presented the Kirigami Haptic Swatches which enables the design of custom haptic feedback elements using classic kirigami and origami mechanisms. Our swatches provide design patterns for linear feedback, bistable feedback, snap-through feedback, and rotational, bistable feedback. We characterized the parameters that affect the form factor as well as the force feedback curve for each of these designs. Using this information, we created custom software for generating cut and fold patterns based on desired form and force inputs. Finally, we shared some example applications and scenarios of how these kirigami and origami buttons can be utilized.

We envision our Kirigami Haptic Swatches to be a useful tool that enables designers and engineers to quickly design and build interactive devices based on their unique, desired haptic feedback characteristics.

\section{ACKNOWLEDGMENTS}

This work was supported by JST ERATO Grant Number JPMJER1501. 


\section{REFERENCES}

[1] Byoungkwon An, Ye Tao, Jianzhe Gu, Tingyu Cheng, Xiang 'Anthony' Chen, Xiaoxiao Zhang, Wei Zhao, Youngwook Do, Shigeo Takahashi, Hsiang-Yun Wu, Teng Zhang, and Lining Yao. 2018. Thermorph: Democratizing 4D Printing of Self-Folding Materials and Interfaces. In Proceedings of the 2018 CHI Conference on Human Factors in Computing Systems (CHI '18). ACM, New York, NY, USA, Article 260, 12 pages. DOI : http://dx.doi.org/10.1145/3173574.3173834

[2] Sean Follmer, Daniel Leithinger, Alex Olwal, Akimitsu Hogge, and Hiroshi Ishii. 2013. inFORM: Dynamic Physical Affordances and Constraints through Shape and Object Actuation. In Proceedings of the 26th Annual ACM Symposium on User Interface Software and Technology (UIST '13). ACM, New York, NY, USA, 417-426. DOI :

http://dx.doi.org/10.1145/2501988.2502032

[3] Christian Frisson, Julien Decaudin, Thomas Pietrzak, Alexander Ng, Pauline Poncet, Fabrice Casset, Antoine Latour, and Stephen A. Brewster. 2017. Designing Vibrotactile Widgets with Printed Actuators and Sensors. In Adjunct Publication of the 30th Annual ACM Symposium on User Interface Software and Technology (UIST '17). ACM, New York, NY, USA, 11-13. DOI : http://dx.doi.org/10.1145/3131785.3131800

[4] Daniel Groeger, Martin Feick, Anusha Withana, and Jürgen Steimle. 2019. Tactlets: Adding Tactile Feedback to 3D Objects Using Custom Printed Controls. In Proceedings of the 32nd Annual ACM Symposium on User Interface Software and Technology (UIST '19). ACM, New York, NY, USA, 923-936. DOI : http://dx.doi.org/10.1145/3332165.3347937

[5] Daniel Groeger and Jürgen Steimle. 2018. ObjectSkin: Augmenting Everyday Objects with Hydroprinted Touch Sensors and Displays. Proc. ACM Interact. Mob. Wearable Ubiquitous Technol. 1, 4, Article 134 (Jan. 2018), 23 pages. DOI:

http://dx.doi.org/10.1145/3161165

[6] Jianzhe Gu, David E. Breen, Jenny Hu, Lifeng Zhu, Ye Tao, Tyson Van de Zande, Guanyun Wang, Yongjie Jessica Zhang, and Lining Yao. 2019. Geodesy: Self-rising 2.5D Tiles by Printing Along 2D Geodesic Closed Path. In Proceedings of the 2019 CHI Conference on Human Factors in Computing Systems (CHI '19). ACM, New York, NY, USA, Article 37, 10 pages. DOI: http://dx.doi.org/10.1145/3290605.3300267

[7] Brandon H. Hanna, Spencer P. Magleby, Robert J. Lang, and Larry L. Howell. 2015. Force-Deflection Modeling for Generalized Origami Waterbomb-Base Mechanisms. Journal of Applied Mechanics 82, 8 (Aug 2015). DOI : http://dx.doi.org/10.1115/1.4030659

[8] Steve Hodges, Nicolas Villar, Nicholas Chen, Tushar Chugh, Jie Qi, Diana Nowacka, and Yoshihiro Kawahara. 2014. Circuit Stickers: Peel-and-stick
Construction of Interactive Electronic Prototypes. In Proceedings of the SIGCHI Conference on Human Factors in Computing Systems (CHI '14). ACM, New York, NY, USA, 1743-1746. DOI : http://dx.doi.org/10.1145/2556288.2557150

[9] Hiroshi Ishii, Daniel Leithinger, Sean Follmer, Amit Zoran, Philipp Schoessler, and Jared Counts. 2015. TRANSFORM: Embodiment of "Radical Atoms" at Milano Design Week. In Proceedings of the 33rd Annual ACM Conference Extended Abstracts on Human Factors in Computing Systems (CHI EA '15). ACM, New York, NY, USA, 687-694. DOI :

http://dx.doi.org/10.1145/2702613.2702969

[10] Sungjune Jang, Lawrence H. Kim, Kesler Tanner, Hiroshi Ishii, and Sean Follmer. 2016. Haptic Edge Display for Mobile Tactile Interaction. In Proceedings of the 2016 CHI Conference on Human Factors in Computing Systems (CHI '16). ACM, New York, NY, USA, 3706-3716. DOI :

http://dx.doi.org/10.1145/2858036.2858264

[11] Mustafa Emre Karagozler, Ivan Poupyrev, Gary K Fedder, and Yuri Suzuki. 2013. Paper Generators: Harvesting Energy from Touching, Rubbing and Sliding. In Proceedings of the 26th Annual ACM Symposium on User Interface Software and Technology (UIST '13). ACM, New York, NY, USA, 23-30. DOI : http://dx.doi.org/10.1145/2501988.2502054

[12] Kunihiro Kato, Hiroki Ishizuka, Hiroyuki Kajimoto, and Homei Miyashita. 2018. Double-Sided Printed Tactile Display with Electro Stimuli and Electrostatic Forces and Its Assessment. In Proceedings of the 2018 CHI Conference on Human Factors in Computing Systems (CHI '18). ACM, New York, NY, USA, Article 450, 12 pages. DOI:http://dx.doi .org/10.1145/3173574.3174024

[13] Kunihiro Kato and Homei Miyashita. 2015. ExtensionSticker: A Proposal for a Striped Pattern Sticker to Extend Touch Interfaces and Its Assessment. In Proceedings of the 33rd Annual ACM Conference on Human Factors in Computing Systems (CHI '15). ACM, New York, NY, USA, 1851-1854. DOI : http://dx.doi.org/10.1145/2702123.2702500

[14] Yoshihiro Kawahara, Steve Hodges, Benjamin S. Cook, Cheng Zhang, and Gregory D. Abowd. 2013. Instant Inkjet Circuits: Lab-based Inkjet Printing to Support Rapid Prototyping of UbiComp Devices. In Proceedings of the 2013 ACM International Joint Conference on Pervasive and Ubiquitous Computing (UbiComp '13). ACM, New York, NY, USA, 363-372. DOI : http://dx.doi.org/10.1145/2493432.2493486

[15] Sunjun Kim and Geehyuk Lee. 2013. Haptic Feedback Design for a Virtual Button along Force-Displacement Curves. In Proceedings of the 26th Annual ACM Symposium on User Interface Software and Technology (UIST '13). ACM, New York, NY, USA, 91-96. DOI : http://dx.doi.org/10.1145/2501988.2502041 
[16] Konstantin Klamka and Raimund Dachselt. 2018. Pushables: A DIY Approach for Fabricating Customizable and Self-Contained Tactile Membrane Dome Switches. In The 31st Annual ACM Symposium on User Interface Software and Technology Adjunct Proceedings (UIST'18 Adjunct). ACM, New York, NY, USA, 1-4. DOI :

http://dx.doi.org/10.1145/3266037. 3266082

[17] Biruta Kresling. 2008. Natural twist buckling in shells: From the hawkmoth's bellows to the deployable kresling-pattern and cylindrical miuraori. In Proceedings of the 6th International Conference on Computation of Shell and Spatial Structures, John F. Abel and J. Robert Cooke, eds., Ithaca, Vol. 11. 12-32.

[18] Yi-Chi Liao, Sunjun Kim, and Antti Oulasvirta. 2018. One Button to Rule Them All: Rendering Arbitrary Force-Displacement Curves. In The 31st Annual ACM Symposium on User Interface Software and Technology Adjunct Proceedings (UIST'18 Adjunct). ACM, New York, NY, USA, 111-113. DOI : http://dx.doi.org/10.1145/3266037.3266118

[19] Yoshinobu Miyamoto. 2015. Rotational Erection System (RES): Origami Extended with Cuts. Origami6 II: Technology Art, Education, Miura K (ed.), AMS (2015), 537-544.

[20] Ken Nakagaki, Luke Vink, Jared Counts, Daniel Windham, Daniel Leithinger, Sean Follmer, and Hiroshi Ishii. 2016. Materiable: Rendering Dynamic Material Properties in Response to Direct Physical Touch with Shape Changing Interfaces. In Proceedings of the 2016 CHI Conference on Human Factors in Computing Systems (CHI'16). ACM, New York, NY, USA, 2764-2772. DOI :

http://dx.doi .org/10.1145/2858036.2858104

[21] Koya Narumi, Steve Hodges, and Yoshihiro Kawahara. 2015. ConductAR: An Augmented Reality Based Tool for Iterative Design of Conductive Ink Circuits. In Proceedings of the 2015 ACM International Joint Conference on Pervasive and Ubiquitous Computing
(UbiComp '15). ACM, New York, NY, USA, 791-800. DOI : http://dx.doi .org/10.1145/2750858.2804267

[22] Masa Ogata. 2018. Magneto-Haptics: Embedding Magnetic Force Feedback for Physical Interactions. In Proceedings of the 31st Annual ACM Symposium on User Interface Software and Technology (UIST '18). ACM, New York, NY, USA, 737-743. DOI : http://dx.doi.org/10.1145/3242587.3242615

[23] Fuminori Okuya, Takuya Umedachi, Kazuo Saito, and Yoshihiro Kawahara. 2018. Crawling Cylindrical Origami Robot Driven by Single Actuator. Origami 7: Proceedings from the seventh meeting of Origami, Science, Mathematics and Education 3 (Sept. 2018), 949-964.

[24] Jie Qi and Leah Buechley. 2014. Sketching in Circuits: Designing and Building Electronics on Paper. In Proceedings of the SIGCHI Conference on Human Factors in Computing Systems (CHI'14). Association for Computing Machinery, New York, NY, USA, 1713-1722. DOI :

http://dx.doi.org/10.1145/2556288.2557391

[25] Austin Reid, Frederic Lechenault, Sergio Rica, and Mokhtar Adda-Bedia. 2017. Geometry and design of origami bellows with tunable response. Phys. Rev. E 95 (Jan 2017), 013002. Issue 1. DOI: http://dx.doi.org/10.1103/PhysRevE.95.013002

[26] Michael Wessely, Theophanis Tsandilas, and Wendy E. Mackay. 2018. Shape-Aware Material: Interactive Fabrication with ShapeMe. In Proceedings of the 31st Annual ACM Symposium on User Interface Software and Technology (UIST '18). ACM, New York, NY, USA, 127-139. DOI :

http://dx.doi.org/10.1145/3242587.3242619

[27] Hiromi Yasuda, Tomohiro Tachi, Mia Lee, and Jinkyu Yang. 2017. Origami-based tunable truss structures for non-volatile mechanical memory operation. Nature Communications 8, 1, Article 962 (Oct. 2017), 7 pages. DOI : http://dx.doi .org/10.1038/s41467-017-00670-w 